\title{
Association Between Leaf Photosynthesis and Biomass Accumulation in Rice Illustrated by $A$ Comprehensive Gas Exchange Profile Across the Growing Season
}

\section{Sotaro Honda}

Graduate School of Agriculture, Tokyo University of Agriculture and Technology

\section{Satoshi Ohkubo}

Graduate School of Agriculture, Tokyo University of Agriculture and Technology

\section{Nan San}

Graduate School of Agriculture, Tokyo University of Agriculture and Technology

\section{Anothai Nakkasame}

Graduate School of Agriculture, Tokyo University of Agriculture and Technology

\section{Kazuki Tomisawa}

Graduate School of Agriculture, Tokyo University of Agriculture and Technology

\section{Keisuke Katsura}

Graduate School of Agriculture, Tokyo University of Agriculture and Technology

\section{Taiichiro Ookawa}

Graduate School of Agriculture, Tokyo University of Agriculture and Technology

\section{Atsushi Nagano}

Faculty of Agriculture, Ryukoku University

Shunsuke Adachi ( $\nabla$ shunsuke.adachi.0210@vc.ibaraki.ac.jp )

College of Agriculture, Ibaraki University

\section{Research Article}

Keywords: biomass, crop growth rate, photosynthesis, inbred lines, rice

Posted Date: December 11th, 2020

DOl: https://doi.org/10.21203/rs.3.rs-122309/v1

License: (c) (1) This work is licensed under a Creative Commons Attribution 4.0 International License. Read Full License 
Version of Record: A version of this preprint was published at Scientific Reports on April 7th, 2021. See the published version at https://doi.org/10.1038/s41598-021-86983-9. 


\section{Abstract}

Leaf photosynthetic rate changes across the growing season as crop plants age. Most studies of leaf photosynthesis focus on a specific growth stage, leaving the question of which pattern of photosynthetic dynamics maximizes crop productivity unanswered. Here we obtained high-frequency data of canopy leaf $\mathrm{CO}_{2}$ assimilation rate $(A)$ of two elite rice (Oryza sativa) cultivars and 76 inbred lines across the whole growing season. The integrated $A$ value after heading was closely associated with crop growth rate (CGR) from heading to harvest, but that before heading was not. A curve-smoothing analysis of $A$ after heading showed that accumulated $A$ at $>80 \%$ of its maximum $\left(A_{80}\right)$ was closely correlated with CGR in analyses of all lines mixed and of lines grouped by genetic background, while the maximum $A$ and accumulated $A$ at $\leq 80 \%$ were less strongly correlated with CGR. We also found a genomic region that may enhance both $A_{80}$ and aboveground biomass at harvest. We propose that maintaining a high $A$ after heading, rather than having high maximum $A$, is a potential target for enhancing rice biomass accumulation.

\section{Introduction}

Rice (Oryza sativa) is one of the most important cereal crops worldwide. To meet the increasing demand for grain as the world's population increases, rice productivity must be increased by $\sim 50 \%$ relative to the current level by $2050^{1,2}$. The rice yield increases during the "green revolution" depended largely on the development of semi-dwarf cultivars with greater harvest index and on greatly increased $\mathrm{N}$ fertilizer application ${ }^{3,4}$. This strategy is reaching its limits, however, because harvest index is reaching its theoretical maximum and excess application of $\mathrm{N}$ fertilizer causes environmental pollution ${ }^{5-7}$. Further enhancement of grain yield must be achieved through increases of total biomass accumulation via improved radiation use efficiency without increased nutrient inputs ${ }^{8}$. Single-leaf photosynthesis has long been considered a target trait for increasing radiation use efficiency $6,9,10$. Recent studies have shown the importance of enhancing single-leaf photosynthesis and crop productivity in the field ${ }^{11}$; for example, the promoted recovery from photoprotection increased biomass production in tobacco (Nicotiana tabacum $)^{12}$, and overproduction of ribulose-1,5-bisphosphate carboxylase/oxygenase (Rubisco) increased grain yield in rice ${ }^{13}$.

Using natural genetic resources could be a useful approach for improving photosynthesis ${ }^{14-16}$. Wide intraspecific variation in net $\mathrm{CO}_{2}$ assimilation rate per leaf area $(A)$ has been found in several crop species, including rice ${ }^{17-19}$ and wheat (Triticum aestivum) ${ }^{20,21}$. The underlying genetic variations can be used in quantitative genetic analyses to identify genomic regions relating to leaf photosynthesis, facilitating DNA marker-assisted selection ${ }^{14,16}$. An important question in such an approach is whether the enhanced $A$ effectively increases total biomass production and grain yield ${ }^{22}$. Positive close correlations of $A$ with plant (or crop) growth rate, biomass production, and final yield through large-scale surveys of diverse sets of accessions have been reported in rice $19,23,24$, wheat ${ }^{25,26}$ and soybean (Glycine max) ${ }^{27}$. 
Simulation analyses showed that a $25 \%$ increase in single-leaf photosynthesis based on rice genetic resources could enhance biomass production by $22-29 \%{ }^{28}$. Furthermore, newer rice cultivars developed in Japan with high yield capacity have higher $A$ than older cultivars, especially after heading ${ }^{29,30}$. These studies underpin the potential for enhanced productivity by improved photosynthesis achieved through the use of natural genetic resources.

In contrast, there are conflicting results on the photosynthesis-productivity relationship. Poor correlations between $A$ and biomass accumulation have been reported in rice ${ }^{18,31}$, wheat ${ }^{21}$ and maize (Zea mays) ${ }^{32}$. Evans (1993) questioned the effects of the genetic improvement of single-leaf photosynthesis for better crop yields ${ }^{33}$. In fact, crop breeding has often selected increased leaf area production at the expense of photosynthetic capacity, as occurred in wheat ${ }^{34}$. The inconsistencies between studies could reduce the potential value of natural genetic resources for improving leaf photosynthesis and delay the enhancement of crop productivity.

The value of $A$ changes across the growing season owing to the progression of plant age and leaf senescence ${ }^{35-37}$. However, most studies of the photosynthesis-productivity relationship selected only one or two growth stages for evaluation of photosynthesis ${ }^{18,19,26,30}$. Such a "snapshot" analysis can reveal only limited aspects of crop production and potentially cause inconsistent results. The need for comprehensive evaluation is supported by the fact that the total $\mathrm{CO}_{2}$ uptake per tobacco plant, calculated from multiple measurements of leaves at several positions throughout the day and the growing season, agreed well with actual dry weight increase ${ }^{38}$. Therefore, multiple photosynthetic measurements are necessary when we examine natural genetic resources across their growing season.

Conventional open gas exchange systems require several to tens of minutes to acclimatize a leaf to the leaf chamber, limiting the number of samples to be examined ${ }^{39}$. To overcome this limitation, we recently created a new closed gas exchange system (MIC-100; Masa International Corporation, Kyoto, Japan), which takes 15-20 s per measurement, 90\% less than conventional open gas exchange systems. We hypothesize that with the new measurement system, tracing photosynthetic dynamics of multiple rice accessions across their growing season will tell us which photosynthetic dynamics can maximize productivity and which developmental stage should be targeted in breeding for photosynthesis.

In previous studies, we determined that the indica cultivar Takanari, which has one of the highest grain yields among Japanese rice cultivars, accumulated more biomass than Nipponbare and Koshihikari, standard japonica cultivars ${ }^{40,41}$. Since then, Takanari has been widely used to analyse the physiological and molecular mechanisms of biomass accumulation ${ }^{42-48}$ and their effects on grain yield ${ }^{49-52}$. Although the higher biomass accumulation in Takanari is characterized by a higher net assimilation rate around the full heading stage, which could be partly explained by the higher leaf photosynthetic capacity, only rough analysis of gas exchange during growth has been conducted ${ }^{41}$. Here, we aimed at collecting the data on temporal changes in canopy photosynthesis of Koshihikari and Takanari over the entire growing season by using the MIC-100 to analyse its association with crop growth rate (CGR) and total 
biomass accumulation. We assumed that photosynthesis in the uppermost fully expanded leaf is representative of canopy photosynthesis, since it has the highest photosynthetic capacity and receives the strongest radiation in the canopy ${ }^{41,44,53}$. We also observed ontogenic changes of chlorophyll content (SPAD value) and single leaf area (single LA). To analyse the phenotypic variation caused by introgressions between the cultivars, we used reciprocal sets of chromosome segment substitution lines (reciprocal CSSLs) derived from a Koshihikari/Takanari cross ${ }^{50,54}$. Each CSSL carries a single genomic segment from the donor cultivar (either Koshihikari or Takanari) in the genetic background of the other cultivar, and the full set of substituted segments covers the entire genome ${ }^{50,55}$. The variation in flowering date is much smaller in CSSLs than in other populations such as recombinant inbred lines, which is advantageous in examining whether changes in photosynthesis affect biomass accumulation. From this study, we propose that maintaining a high rate of photosynthesis after heading, rather than having a high maximum photosynthetic rate, can increase total biomass accumulation.

\section{Results}

\section{Ontogenic changes in photosynthesis and biomass accumulation}

We divided the growth period into Phase I-from transplanting to the first biomass sampling (at heading) -and Phase II-from the first sampling to the second sampling (at harvest) (Fig. 1). (See days to heading [DTH] data of all rice lines in Supplementary dataset.) As a general trend, $A$ reached the maximum at around 30-35 days after transplanting (DAT) and then gradually decreased over time (Fig. 1a). During Phase I, $A$ values of Takanari-background CSSLs and Takanari (Takanari lines) tended to be lower than those of Koshihikari-background CSSLs and Koshihikari (Koshihikari lines). During Phase II, $A$ values of Takanari lines remained higher than those of Koshihikari lines (Fig. 1a). SPAD values showed a similar trend (Fig. 1b). Single LA gradually increased with crop growth and reached a maximum at around 65 DAT in Koshihikari lines and 72 DAT in Takanari lines (Fig. 1c). Single LA of Takanari lines was larger than that of Koshihikari lines during Phase I, and larger still during Phase II (Fig. 1C).

Integrated $A\left(A_{\text {int }}\right)$, the apparent total $\mathrm{CO}_{2}$ uptake calculated by sum of trapezoidal area under each pair of adjacent measurements, was $10 \%$ lower in Takanari lines than in Koshihikari lines during Phase I, but was $23 \%$ higher during Phase II ( $P<0.001$; Fig. 2a). Mean single LA was significantly higher in Takanari lines than in Koshihikari lines during Phase I, and even higher during Phase II $(P<0.001 ;$ Fig. 2b). There was no significant difference in aboveground biomass at the first sampling ( $\left.A G B_{1}\right)$ ) or in CGR during Phase I (CGR Phase I) between Koshihikari lines and Takanari lines, while AGB at the second sampling (AGB ॥) and CGR during Phase II (CGR Phase II) were significantly higher in Takanari lines than in Koshihikari lines, by $25 \%$ and $40 \%$, respectively $(P<0.001$; Fig. 2c, d). The standard deviation (SD) in each background was larger during Phase II than during Phase I (for instance, for CGR in Koshihikari lines: 0.72 during Phase I but 2.51 during Phase II; Table S1). These results indicate that the genetic differences between Koshihikari and Takanari and between lines of each genetic background were more notable 
during Phase II than during Phase I. The AGB ॥ was closely correlated with CGR Phase II $(r=0.97)$, not with CGR Phase I $\left(r=0.34\right.$; Supplementary Fig. S4). CGR Phase I was not correlated with $A_{\text {int }}$ during Phase I ( $A_{\text {int }}$ Phase I $)(r=-0.10)$ and was only slightly correlated with mean single LA during Phase I ( $\left.L A_{\text {mean Phase } \mathrm{I}}\right)(r=$ 0.28 ), while CGR Phase II was strongly correlated with these values ( $r=0.75$ for $A_{\text {int }}$ during Phase II [ $A_{\text {int }}$

Phase II], $r=0.82$ for mean single LA during Phase II [LA mean Phase II]; Supplementary Fig. S4). These results indicate that $A G B$ ॥ depends largely on CGR Phase ॥, which in turn is correlated closely with photosynthesis and single LA during Phase II.

\section{Curve-smoothing analysis during Phase II and associations of parameters with crop growth rate}

For detailed analysis of photosynthetic dynamics during Phase II, we applied curve-smoothing analysis to the $A$ and SPAD values (Fig. 3a, d). Both curves were upward-convex, peaking several days after beginning of Phase II, and decreased over time. The total area under the curve $\left(A_{\text {all }}\right)$ and the maximum $A$ $\left(A_{\max }\right)$ were higher in Takanari than in Koshihikari, by around $26 \%$ each (Fig. 3b, c). When $A_{\text {all }}$ was divided into accumulated $A$ at $>80 \%$ of $A_{\max }\left(A_{80}\right)$ and accumulated $A$ at $\leq 80 \%$ of $A_{\max }\left(A_{\text {dec }}\right)$ at $D_{\text {onset }}(1$ day before $A$ declined below $80 \%$ of $A_{\text {max }}$ ), Takanari had a higher $A_{80}$ than Koshihikari but a similar $A_{\text {dec- }}$. Takanari also had higher values of $\mathrm{SPAD}_{80}$ and $S P A D_{\text {dec }}$ (the mean SPAD values of the two phases divided at $D_{\text {onset }}$ ) than Koshihikari (Fig. 3e, f). The values of all CSSLs are shown in the Supplementary dataset.

The correlations between biomass accumulation and photosynthetic parameters after heading showed that CGR Phase II was closely correlated with $A_{\text {all }}$ (Fig. 4). In turn, $A_{\text {all }}$ was closely correlated with $A_{\text {max }}, A_{80}$ and $D_{\text {onset, }}$ and was moderately negatively correlated with $A_{\text {dec }}$. These results suggest that $A_{\text {all }}$ is determined mainly by $A_{80}$, the magnitude of which can be explained by both $A_{\text {max }}$ and $D_{\text {onset }}$. CGR Phase II

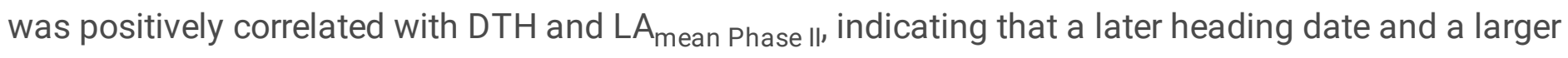
single LA could enhance biomass accumulation. SPAD 80 was positively correlated with $A_{80}$, but SPAD dec was not correlated with $A_{\text {dec }}$.

\section{Analysis by genetic background}

The results of the above analyses should be affected considerably by the genetic background, because the Takanari lines had consistently higher values of most parameters after heading. So we conducted separate analyses by genetic background. AGB „| was closely correlated with CGR Phase ॥ in each background ( $r=0.94$ for Koshihikari lines, $r=0.88$ for Takanari lines; Supplementary Fig. S5). CGR Phase II was not correlated with $A_{\max }$ in either background ( $r=-0.12$ for Koshihikari lines, $r=0.16$ for Takanari lines), but it was significantly correlated with $A_{80}$ ( $r=0.31$ for Koshihikari lines; $r=0.43$ for Takanari lines) and with $D_{\text {onset }}(r=0.43$ for Koshihikari lines; Supplementary Fig. S5, Fig. 5a-c). The association between CGR Phase II and $D_{\text {onset }}$ in Takanari lines was close to significant $(r=0.28, P=0.091$; Supplementary Fig. S5b, Fig. 5c). These results indicate that maintaining a high rate of photosynthesis for longer, rather than 
having a higher $A_{\max }$, was related to higher biomass accumulation during Phase II in each background. We also found a significant relationship between CGR Phase II and LA mean Phase II in each background ( $r=$ 0.44 for Koshihikari lines, $r=0.49$ for Takanari lines; Supplementary Fig. S5, Fig. 5d). The factors affecting total biomass accumulation are presented in Fig. 5e. By multiple linear regression analysis, the combined contribution of $A_{80}$ and LA mean Phase II to CGR Phase II variation was $25 \%$ for Koshihikari lines and $31 \%$ for Takanari lines.

Among the CSSLs, $A_{80}$ values of SL1212 and SL1310, with a single genomic segment on chromosome 3 from the introgression parent, were, respectively, 11\% higher than that of Koshihikari and $10 \%$ lower than that of Takanari (Supplementary Fig. S6a, Fig. 5b). AGB ॥ and CGR Phase ॥ of SL1212 were 13\% and 13\%, respectively, higher than those of Koshihikari, and those of SL1310 were 14\% and 28\% lower than those of Takanari (Supplementary Fig. S6b, c). These results suggest that genes on the genomic segment of chromosome 3 regulate both photosynthesis and total biomass accumulation.

\section{Discussion}

Improving leaf photosynthetic capacity has long been considered a promising target to increase biomass production and yield in crop species ${ }^{6,9,10}$. However, poor correlations between leaf photosynthetic rate and biomass accumulation or yield have been reported, perhaps in part owing to limited datasets $^{18,21,31,32,56}$. To understand the association of photosynthetic rate and biomass accumulation across the entire growing season, we obtained high-frequency data of $A$, SPAD and single LA of the canopy leaf and tested correlations with CGR using reciprocal CSSLs and their parental cultivars.

During Phase I (transplanting to heading), differences in leaf photosynthesis had little effect on biomass production. Takanari lines had lower $A$ and SPAD values and larger single LA than Koshihikari lines (Figs. 1, 2). AGB , and CGR Phase I did not differ between Koshihikari and Takanari lines (Fig. 2), which can be explained by the offset of the lower $A$ by the larger single LA in Takanari lines. Taylaran et al. (2011) likewise showed that Takanari had a similar plant growth rate to Koshihikari during the vegetative stage owing to its lower net assimilation rate but the higher mean leaf area per plant ${ }^{41}$. We also found a smaller variation in these traits among lines of each background (41 Koshihikari lines, 37 Takanari lines) during Phase I than during Phase II (Fig. 2; Supplementary Table S1), which suggests that the genomic introgressions between the cultivars have little effect on phenotypic expression before heading. In contrast, a wide genetic variation in biomass accumulation (227\%) among 204 global mini-core accessions and 11 elite Chinese rice cultivars at the mid-vegetative stage (60 days after emergence) was reported ${ }^{19}$. The authors also showed that $A$ under low light was highly related to biomass accumulation, suggesting that simultaneous improvements of photosynthetic rate and biomass accumulation during early growth can be achieved by using a diverse set of germplasms ${ }^{19}$. 
During Phase II (heading to harvest), photosynthetic parameters were closely associated with biomass accumulation. The $A$ value of Takanari lines increased and remained higher than that of Koshihikari lines

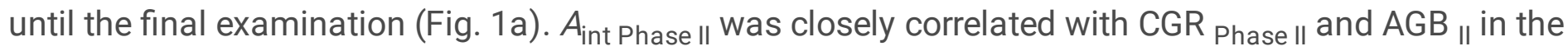
analysis of all datasets combined (Supplementary Fig. S4). In the separate analysis of each background, CGR Phase II and $A_{80}$ were significantly correlated in both Koshihikari and Takanari lines (Supplementary Fig. S5). These results suggest that higher photosynthetic performance after heading would enhance biomass accumulation. Interestingly, $A_{\max }$ was not correlated with CGR phase II in either Koshihikari or Takanari lines (Fig. 5a, Supplementary fig. S5). In addition, $A_{\max }$ was not correlated with $A_{80}$ in Takanari lines, although it was significantly correlated in Koshihikari lines (Supplementary Fig. S5), suggesting that increasing $A_{\max }$ is not always an efficient strategy for enhancing biomass accumulation. Many physiological and molecular analyses have focused on the maximum photosynthetic rate of the flag leaf on the assumption that it has the highest photosynthetic activity in the crop canopy after heading, which would be closely correlated with biomass accumulation and yield $26,42,54,57,58$. However, our results show that maintaining a high rate of photosynthesis after heading, rather than having a high $A_{\text {max }}$, is more closely associated with biomass accumulation. We identified a genomic region that may simultaneously increase (or decrease) $A_{80}$ and CGR Phase ॥ without increasing $A_{\max }$ (Fig. 5, Supplementary Fig. S6). We propose that as limited evaluation of photosynthesis could select lines with poor biomass accumulation, ontogenic changes in photosynthesis after heading should be examined for simultaneously enhancing photosynthetic performance and biomass accumulation.

The variation in $\mathrm{LA}_{\text {mean }}$ Phase II was also associated with the variation in CGR Phase II in both the combined analysis of all lines and the separate analyses of Koshihikari and Takanari lines (Figs. 4, 5d, Supplementary Fig. S5). This suggests that breeding for the selection of plants with larger flag leaves may enhance the total biomass accumulation. However, very large leaves and too many tillers could reduce sunlight penetration into the canopy, thereby increasing canopy respiration rate and decreasing the total biomass accumulation ${ }^{59}$. This issue may not be obvious in our results, but it should be considered in different growth environments.

Although we found significant correlations of $A_{80}$ and LA mean Phase II with CGR Phase I, the combined contribution of $A_{80}$ and LA mean Phase II to CGR Phase II variation was only $25-31 \%$. This indicates the presence of other major determinants behind the variation in CGR. The first possibility is tiller growth: active tillering increases the total leaf area of a plant, greatly contributing to total biomass accumulation and panicle number ${ }^{60}$. Although we did not examine tiller numbers here, panicle number varies widely among these $\mathrm{CSSLs}^{50}$. The second possibility is the photosynthetic capacity and single LA of leaves at lower positions. A recent study showed that the balance of photosynthetic capacity between the flag leaf and the leaf immediately below it has significant effects on canopy photosynthesis in wheat ${ }^{61}$. The third possibility is the degree of light penetration to the bottom of the canopy. Better light penetration, which is achieved by large leaf inclination angles and decreased chlorophyll content of the canopy leaves, can maximize canopy photosynthesis ${ }^{6,62}$. Takanari has one of the highest leaf inclination angles among rice 
cultivars, which is considered an important determinant of its higher biomass accumulation ${ }^{63}$. The fourth possibility is adaptation to the environment, especially light and vapour pressure deficit. Sunlight reaching the leaf surface fluctuates on the order of minutes to seconds owing to cloud, wind and selfshading ${ }^{64}$. The time-lag inherent in reaching a new steady-state rate of photosynthesis after a fluctuation would diminish the total carbon gain ${ }^{65,66}$. The photosynthetic rate can decrease in the afternoon on sunny days with high vapour pressure deficit, so-called "midday depression", largely because of closed stomata and photoinhibition ${ }^{3,67}$. So we need a comprehensive simulation model using these complex physiological factors and the association of the underlying genomic regions to explain the difference in biomass accumulation among lines. Our high-frequency datasets of photosynthesis may contribute to the development of such a model.

In conclusion, maintaining a higher photosynthetic rate, rather than achieving the maximum rate, after heading was closely associated with biomass accumulation. We identified a genomic region likely to simultaneously increase $A_{80}$ and biomass accumulation, although further investigation is necessary. We propose that examination of the dynamics of photosynthesis throughout the entire growing period is important to the use of natural genetic resources for breeding selection. In contrast, the limited contribution of $A_{80}$ to biomass production suggests essential roles of other physiological factors in biomass variation. A comprehensive model explaining the role of genetic variation in biomass production by multiple physiological properties and the roles of key genes is required.

\section{Methods}

\section{Plant cultivation}

We grew cultivars Koshihikari and Takanari and reciprocal sets of CSSLs (41 lines in Koshihikari background, 39 lines in Takanari background) ${ }^{50}$. Lines SL1208, SL1335 and SL1336 had a dwarf plant structure, probably due to hybrid breakdown associated with the interaction of $h b d 2$ and $h b d 3$, and SL1320 did not produce panicles during the experiment, probably owing to the inserted $\mathrm{Hd} 1 \mathrm{gene} \mathrm{e}^{50}$. We excluded these four lines from our analyses. Seeds were sown in plastic cups filled with artificial soil on 7 May 2019, and the seedlings were grown until the fourth to fifth leaf stage in the greenhouse. They were transplanted into a paddy field (an alluvial clay loam) of Tokyo University of Agriculture and Technology $\left(35^{\circ} 39^{\prime} \mathrm{N}, 139^{\circ} 28^{\prime} \mathrm{E}\right)$ on 22 May with a basal dressing of inorganic fertilizer supplying $30 \mathrm{~kg} \mathrm{~N}, 60 \mathrm{~kg} \mathrm{P}$, and $60 \mathrm{~kg} \mathrm{~K} \mathrm{ha}^{-1}$. One-third of the total $\mathrm{N}$ was applied as ammonium sulphate, and the other two-thirds as slow-release urea (LP-50 \& LPS-100; JCAM Agri Co., Ltd, Tokyo, Japan). No topdressing was applied. The plant density was $22.2 \mathrm{~m}^{-2}$ (at a spacing of $30 \mathrm{~cm} \times 15 \mathrm{~cm}$ ) with one plant per hill, and plants were grown under submerged conditions. Each line was grown in three replicate plots in 2 rows of 20 plants $(60 \mathrm{~cm} \times 300 \mathrm{~cm})$. Plots were randomized, but lines of each background group were planted adjacent (Supplementary Fig. S1).

\section{Phenotypic analysis}


The uppermost newly expanded leaf on the main tiller of one plant per plot was used for phenotypic analyses. The net $\mathrm{CO}_{2}$ assimilation rate was measured with a closed-type portable photosynthesis system (MIC-100; Masa International Corporation, Kyoto, Japan; https://www.weather.co.jp/ catalog_html/MIC-100.html), which consists of a console and a chamber head with an aperture area of $2 \mathrm{~cm} \times 3 \mathrm{~cm}$ (Supplementary Fig. S2a). A non-dispersive infrared sensor at the bottom of the chamber measures $\mathrm{CO}_{2}$ concentration every $0.1 \mathrm{~s}$. To prevent rapid inactivation of the leaf's photosynthetic activity, a light-emitting diode lamp at the chamber top supplies a photosynthetic photon flux density of $1200 \mu \mathrm{mol}$ photons $\mathrm{m}^{-2} \mathrm{~s}^{-1}$. After an intact leaf is enclosed in the chamber clip, air flow from the atmosphere is blocked off, and the rate of decrease of $\mathrm{CO}_{2}$ concentration from 400 to $390 \mathrm{ppm}$ is monitored to calculate net $\mathrm{CO}_{2}$ assimilation rate. Each measurement was completed within $20 \mathrm{~s}$. Measurements were taken in sunlight between 08:00 and 13:00 $\mathrm{h}$ on dry days (the solar radiation during measurements was 500-1300 $\mu$ mol photons $\mathrm{m}^{-2} \mathrm{~s}^{-1}$ ). The SPAD value as a proxy for leaf chlorophyll content was measured with a chlorophyll meter (SPAD-502; Konica Minolta, Osaka, Japan; Supplementary Fig. S2b). Leaves were sampled and transported to the laboratory without dehydration. The leaves put in a transparent folder were passed through a commercial document scanner (ScanSnap iX1500; Fujitsu, Kanagawa, Japan; Supplementary Fig. S2c). The single LA and the partial LA in the MIC100 chamber were measured in Image J software (National Institutes of Health, Bethesda, MD, USA). The partial LA was used for the calculation of net $\mathrm{CO}_{2}$ assimilation rate per leaf area $\left(A, \mu \mathrm{mol} \mathrm{m} \mathrm{m}^{-2} \mathrm{~s}^{-1}\right)$. Measurements were conducted once a week from 3 weeks after transplanting (14 June, 23 DAT) to harvest (27 September, 128 DAT), and additional measurements were also conducted around heading and mid-ripening stage (20 days in total). In total, 246 leaves were measured per day, 4632 leaves during the experiment. $A_{\text {int }}$ was calculated by summing the trapezoidal area under each pair of adjacent measurement cycles. Mean single LA was calculated as the average of linear interpolated values.

\section{Sampling of aboveground biomass}

The aboveground biomass was examined at heading (1 August, 71 DAT; AGB ) and harvest (28 September, 129 DAT; AGB „). Eight plants in each plot were sampled and air-dried in a greenhouse until weighing. Air-dried samples of parental plants were dried in a ventilated oven to calculate the water content ratio of the air-dried samples. The biomass accumulation was expressed as dry weight (g) per $\mathrm{m}^{2}$. CGR from transplanting (considered as 0 ) to heading (Phase I) and from heading to harvest (Phase II) were calculated.

\section{Curve smoothing and statistical analysis}

All statistical analyses were performed in R v. 4.0.2 software ${ }^{68}$. The changes in $A$ and SPAD values during Phase II in each rice line were smoothed by the Locally Weighted Scatterplot Smoother (LOESS) algorithm with the smoothing parameter fixed at $1.0^{69}$. We defined $A_{\max }\left(\mu \mathrm{mol} \mathrm{m} \mathrm{m}^{-2} \mathrm{~s}^{-1}\right)$ as the maximum fitted value of $A$, and $D_{\text {onset }}$ (day) as 1 day before $A$ declined below $80 \%$ of $A_{\max }$ (Fig. 3). We also defined $A_{\text {all }}$ ( $\mu \mathrm{mol} \mathrm{m} \mathrm{m}^{-2} \mathrm{~s}^{-1}$ phase $^{-1}$ ) as accumulated $A$ by curve-smoothing during Phase II (from 72 to $128 \mathrm{DAT}$ ), $A_{80}$ 
( $\mu \mathrm{mol} \mathrm{m} \mathrm{m}^{-2} \mathrm{~s}^{-1}$ phase $^{-1}$ ) as accumulated $A$ from 72 DAT to $D_{\text {onset, }}$ and $A_{\text {dec }}\left(\mu \mathrm{mol} \mathrm{m}{ }^{-2} \mathrm{~s}^{-1}\right.$ phase ${ }^{-1}$ ) as accumulated $A$ from $D_{\text {onset }}$ to 128 DAT. We show the changes in correlation coefficients between CGR Phase II and $A_{10}$ to $A_{90}$ (accumulated $A$ at $>10 \%$ to $>90 \%$ of $A_{\text {max }}$ Supplementary Fig. S7). SPAD 80 is the mean SPAD value from 72 DAT to $D_{\text {onset }}$ and SPAD dec is mean SPAD value from $D_{\text {onset }}$ to 128 DAT. Statistical differences were tested by Welch's two-sided $t$-test. Pearson's correlation coefficient was calculated, and the significance of relationships was tested by two-sided $t$-tests.

\section{Abbreviations}




\begin{tabular}{|c|c|}
\hline$A$ & net $\mathrm{CO}_{2}$ assimilation rate per leaf area \\
\hline$A_{80}$ & accumulated $A$ at $>80 \%$ of $A_{\max }$ \\
\hline$A_{\text {all }}$ & accumulated $A$ during Phase II \\
\hline$A_{\mathrm{dec}}$ & accumulated $A$ at $\leq 80 \%$ of $A_{\max }$ \\
\hline $\mathrm{AGB}_{1}$ & aboveground biomass at first sampling \\
\hline $\mathrm{AGB}_{\|}$ & aboveground biomass at second sampling \\
\hline$A_{\text {int }}$ & integrated $A$ \\
\hline$A_{\text {int Phase I }}$ & integrated $A$ during Phase I \\
\hline$A_{\text {int Phase II }}$ & integrated $A$ during Phase II \\
\hline$A_{\max }$ & maximum fitted value of $A$ during Phase II \\
\hline CGR & crop growth rate \\
\hline CGR Phase I & CGR during Phase I \\
\hline CGR Phase II & CGR during Phase II \\
\hline CSSL & chromosome segment substitution line \\
\hline DAT & days after transplanting \\
\hline$D_{\text {onset }}$ & 1 day before $A$ declined below $80 \%$ of $A_{\max }$ \\
\hline DTH & days to heading \\
\hline Koshihikari lines & Koshihikari-background CSSLs and Koshihikari \\
\hline LA & leaf area \\
\hline $\mathrm{LA}_{\text {mean Phase }}$ & mean single LA during Phase I \\
\hline LA $A_{\text {mean Phase II }}$ & mean single LA during Phase II \\
\hline $\mathrm{SPAD}_{80}$ & mean SPAD value before $D_{\text {onset }}$ \\
\hline$S P A D_{d e c}$ & mean SPAD value after $D_{\text {onset }}$ \\
\hline Takanari lines & Takanari-background CSSLs and Takanari \\
\hline
\end{tabular}

\section{Declarations}




\section{Acknowledgements}

This work was supported in part by the Japan Science and Technology Agency, CREST grant number JPMJCR1502 (to S.A. and A.N), and by JSPS KAKENHI (grant numbers JP18K05585 and JP19H02939 to S.A., and JP19H02940 to T.O. and S.A.). We are grateful to Ms. T. Yamanouchi and Ms. Y. Yamashita for assistance with rice cultivation and data analysis.

\section{Author contributions}

A.J.N. and S.A. designed the experiments. S.H., S.O., N.S., A.N., and K.T. performed the experiments. S.H., S.O., and S.A. wrote the manuscript. K.K., T.O., and A.J.N. contributed to finalization of the manuscript.

\section{Competing interests}

The authors declare no competing interests.

\section{Data availability}

All data are available from the corresponding author on reasonable request.

\section{References}

1. Ray, D. K., Mueller, N. D., West, P. C. \& Foley, J. A. Yield trends are insufficient to double global crop production by 2050. PLOS ONE 8, e66428; 10.1371/journal.pone.0066428 (2013).

2. Long, S. P., Marshall-Colon, A. \& Zhu, X.-G. Meeting the global food demand of the future by engineering crop photosynthesis and yield potential. Cell 161, 56-66 (2015).

3. Peng, S. et al. Grain yield of rice cultivars and lines developed in the Philippines since 1966. Crop Sci. 40, 307-314 (2000).

4. Murchie, E. H., Pinto, M. \& Horton, P. Agriculture and the new challenges for photosynthesis research. New Phytol. 181, 532-552 (2009).

5. Mann, C. C. Crop scientists seek a new revolution. Science 283, 310-314 (1999).

6. Long, S. P., Zhu, X.-G., Naidu, S. L. \& Ort, D. R. Can improvement in photosynthesis increase crop yields? Plant Cell Environ. 29, 315-330 (2006).

7. Sage, R. F., Adachi, S. \& Hirasawa, T. Improving photosynthesis in rice: from small steps to giant leaps. in Achieving sustainable cultivation of rice Volume 1: Breeding for higher yield and quality (ed. Sasaki, T.) 99-130 (Burleigh Dodds Science Publishing, 2017).

8. Zhu, X.-G., Long, S. P. \& Ort, D. R. Improving photosynthetic efficiency for greater yield. Ann. Rev. Plant Biol. 61, 235-261 (2010). 
9. Monteith, J. L. \& Moss, C. Climate and the efficiency of crop production in Britain. Phil. Trans. R. Soc. B: Biol. Sci. 281, 277-294 (1977).

10. Furbank, R. T., Sharwood, R., Estavillo, G. M., Silva-Perez, V. \& Condon, A. G. Photons to food: genetic improvement of cereal crop photosynthesis. J. Exp. Bot. 71, 2226-2238 (2020).

11. Simkin, A. J., López-Calcagno, P. E. \& Raines, C. A. Feeding the world: improving photosynthetic efficiency for sustainable crop production. J. Exp. Bot. 70, 1119-1140 (2019).

12. Kromdijk, J. et al. Improving photosynthesis and crop productivity by accelerating recovery from photoprotection. Science 354, 857-861 (2016).

13. Yoon, D.-K. et al. Transgenic rice overproducing Rubisco exhibits increased yields with improved nitrogen-use efficiency in an experimental paddy field. Nature Food 1, 134-139; 10.1038/s43016020-0033-x (2020).

14. Flood, P. J., Harbinson, J. \& Aarts, M. G. M. Natural genetic variation in plant photosynthesis. Trends Plant Sci. 16, 327-335 (2011).

15. Furbank, R. T., Jimenez-Berni, J. A., George-Jaeggli, B., Potgieter, A. B. \& Deery, D. M. Field crop phenomics: enabling breeding for radiation use efficiency and biomass in cereal crops. New Phytol. 223, 1714-1727 (2019).

16. Adachi, S., Ohkubo, S., San, N. S. \& Yamamoto, T. Genetic determination for source capacity to support breeding of high-yielding rice (Oryza sativa). Mol. Breeding 40, 20; 10.1007/s11032-0201101-5 (2020).

17. Kanemura, T., Homma, K., Ohsumi, A., Shiraiwa, T. \& Horie, T. Evaluation of genotypic variation in leaf photosynthetic rate and its associated factors by using rice diversity research set of germplasm. Photosynth. Res. 94, 23-30 (2007).

18. Jahn, C. E. et al. Genetic variation in biomass traits among 20 diverse rice varieties. Plant Physiol. 155, 157-168 (2011).

19. Qu, M. et al. Leaf photosynthetic parameters related to biomass accumulation in a global rice diversity survey. Plant Physiol. 175, 248-258 (2017).

20. Sadras, V., Lawson, C. \& Montoro, A. Photosynthetic traits in Australian wheat varieties released between 1958 and 2007. Field Crops Res. 134, 19-29 (2012).

21. Driever, S. M., Lawson, T., Andralojc, P. J., Raines, C. A. \& Parry, M. A. Natural variation in photosynthetic capacity, growth, and yield in 64 field-grown wheat genotypes. J. Exp. Bot. 65, 49594973 (2014).

22. Peng, S. Single-leaf and canopy photosynthesis of rice in Studies in Plant Science, Volume 7 Redesigning Rice Photosynthesis to Increase Yield (eds. Sheehy, J. E., Mitchell, P. L. \& Hardy, B.) 213228 (Elsevier, 2000).

23. Murata, Y. Studies on photosynthesis in rice plants and its culture significance. Bul. Natl. Inst. Agric. Sci. Series D 9, 1-169 (1961). 
24. Cook, M. G. \& Evans, L. T. Some physiological aspects of the domestication and improvement of rice (Oryza spp.). Field Crops Res. 6, 219-238 (1983).

25 . Fischer, R. et al. Wheat yield progress associated with higher stomatal conductance and photosynthetic rate, and cooler canopies. Crop Sci. 38, 1467-1475 (1998).

26. Carmo-Silva, E. et al. Phenotyping of field-grown wheat in the UK highlights contribution of light response of photosynthesis and flag leaf longevity to grain yield. J. Exp. Bot. 68, 3473-3486 (2017).

27. Buttery, B., Buzzell, R. \& Findlay, W. Relationships among photosynthetic rate, bean yield and other characters in field-grown cultivars of soybean. Can. J. Plant Sci. 61, 190-197 (1981).

28. Gu, J., Yin, X., Stomph, T. J. \& Struik, P. C. Can exploiting natural genetic variation in leaf photosynthesis contribute to increasing rice productivity? A simulation analysis. Plant Cell Environ. 37, 22-34 (2014).

29. Sasaki, H. \& Ishii, R. Cultivar differences in leaf photosynthesis of rice bred in Japan. Photosynth. Res. 32, 139-146 (1992).

30. Zhang, W.-H. \& Kokubun, M. Historical changes in grain yield and photosynthetic rate of rice cultivars released in the 20th century in Tohoku region. Plant Prod. Sci. 7, 36-44 (2004).

31. Murchie, E. H., Yang, J., Hubbart, S., Horton, P. \& Peng, S. Are there associations between grain-filling rate and photosynthesis in the flag leaves of field-grown rice? J. Exp. Bot. 53, 2217-2224 (2002).

32. Crosbie, T. M., Pearce, R. B. \& Mock, J. J. Relationships among $\mathrm{CO}_{2}$-exchange rate and plant traits in lowa Stiff Stalk Synthetic maize population. Crop Sci. 18, 87-90 (1978).

33. Evans, L. T. Crop Evolution, Adaptation and Yield. (Cambridge University Press, 1993).

34. Gifford, R. M. \& Evans, L. T. Photosynthesis, carbon partitioning, and yield. Ann. Rev. Plant Physiol. 32, 485-509 (1981).

35. Rawson, H., Hindmarsh, J., Fischer, R. \& Stockman, Y. Changes in leaf photosynthesis with plant ontogeny and relationships with yield per ear in wheat cultivars and 120 progeny. Funct. Plant Biol. 10, 503-514 (1983).

36. Ohsumi, A. et al. A model explaining genotypic and ontogenetic variation of leaf photosynthetic rate in rice (Oryza sativa) based on leaf nitrogen content and stomatal conductance. Ann. Bot. 99, 265273 (2007).

37. Adachi, S. et al. Fine mapping of carbon assimilation rate 8 , a quantitative trait locus for flag leaf nitrogen content, stomatal conductance and photosynthesis in rice. Front. Plant Sci. 8; 10.3389/fpls.2017.00060 (2017).

38. Peterson, R. B. \& Zelitch, I. Relationship between net $\mathrm{CO}_{2}$ assimilation and dry weight accumulation in field-grown tobacco. Plant Physiol. 70, 677-685 (1982).

39. Salter, W. T., Gilbert, M. E. \& Buckley, T. N. A multiplexed gas exchange system for increased throughput of photosynthetic capacity measurements. Plant Methods 14, 80; 10.1186/s13007-0180347-y (2018). 
40. Xu, Y.-F., Ookawa, T. \& Ishihara, K. Analysis of the photosynthetic characteristics of the high-yielding rice cultivar Takanari. Jpn. J. Crop Sci. 66, 616-623 (1997).

41. Taylaran, R. D., Adachi, S., Ookawa, T., Usuda, H. \& Hirasawa, T. Hydraulic conductance as well as nitrogen accumulation plays a role in the higher rate of leaf photosynthesis of the most productive variety of rice in Japan. J. Exp. Bot. 62, 4067-4077 (2011).

42. Takai, T. et al. A natural variant of $N A L 1$, selected in high-yield rice breeding programs, pleiotropically increases photosynthesis rate. Sci. Rep. 3, 2149; 10.1038/srep02149 (2013).

43. Chen, C. P. et al. Do the rich always become richer? Characterizing the leaf physiological response of the high-yielding rice cultivar Takanari to free-air $\mathrm{CO}_{2}$ enrichment. Plant Cell Physiol. 55, 381-391 (2014).

44. Muryono, M. et al. Nitrogen distribution in leaf canopies of high-yielding rice cultivar Takanari. Crop Sci. 57, 2080-2088 (2017).

45. Takai, T. et al. Effects of yield-related QTLS SPIKE and GPS in two indica rice genetic backgrounds. Plant Prod. Sci. 20, 467-476; 10.1080/1343943X.2017.1385404 (2017).

46. Ikawa, H. et al. Increasing canopy photosynthesis in rice can be achieved without a large increase in water use-A model based on free-air $\mathrm{CO}_{2}$ enrichment. Global Change Biol. 24, 1321-1341 (2018).

47. Adachi, S. et al. High-yielding rice Takanari has superior photosynthetic response to a commercial rice Koshihikari under fluctuating light. J. Exp. Bot. 70, 5287-5297 (2019).

48. Ohkubo, S., Tanaka, Y., Yamori, W. \& Adachi, S. Rice cultivar Takanari has higher photosynthetic performance under fluctuating light than Koshihikari, especially under limited nitrogen supply and elevated $\mathrm{CO}_{2}$. Front. Plant Sci. 11; 10.3389/fpls.2020.01308 (2020).

49. Katsura, K., Okami, M., Mizunuma, H. \& Kato, Y. Radiation use efficiency, N accumulation and biomass production of high-yielding rice in aerobic culture. Field Crops Res. 117, 81-89 (2010).

50. Takai, T. et al. Genetic mechanisms underlying yield potential in the rice high-yielding cultivar Takanari, based on reciprocal chromosome segment substitution lines. BMC Plant Biol. 14, 295; 10.1186/s12870-014-0295-2 (2014).

51. Nakano, H. et al. Quantitative trait loci for large sink capacity enhance rice grain yield under free-air $\mathrm{CO}_{2}$ enrichment conditions. Sci. Rep. 7, 1827; 10.1038/s41598-017-01690-8 (2017).

52. Hasegawa, T. et al. A high-yielding rice cultivar "Takanari" shows no $\mathrm{N}$ constraints on $\mathrm{CO}_{2}$ fertilization. Front. Plant Sci. 10, 361; 10.3389/fpls.2019.00361 (2019).

53. Hirotsu, N. et al. Partial loss-of-function of NAL 1 alters canopy photosynthesis by changing the contribution of upper and lower canopy leaves in rice. Sci. Rep. 7, 15958; 10.1038/s41598-01715886-5 (2017).

54. Adachi, S. et al. Genetic architecture of leaf photosynthesis in rice revealed by different types of reciprocal mapping populations. J. Exp. Bot. 70, 5131-5144 (2019).

55. Yamamoto, T., Uga, Y. \& Yano, M. Genomics-assisted allele mining and its integration into rice breeding in Genomics of Plant Genetic Resources Volume 2 Crop productivity, food security and 
nutritional quality (eds. Tuberosa, R., Graner, A. \& Frison, E.) 251-265 (Springer, 2014).

56. Zelitch, I. The close relationship between net photosynthesis and crop yield. Bioscience 32, 796-802 (1982).

57. Adachi, S. et al. Identification and characterization of genomic regions on chromosomes 4 and 8 that control the rate of photosynthesis in rice leaves. J. Exp. Bot. 62, 1927-1938 (2011).

58. Gu, J., Yin, X., Struik, P. C., Stomph, T. J. \& Wang, H. Using chromosome introgression lines to map quantitative trait loci for photosynthesis parameters in rice (Oryza sativa L.) leaves under drought and well-watered field conditions. J. Exp. Bot. 63, 455-469 (2012).

59. Venkatraman, S., Praveen, K. \& Long, S. P. Decreasing, not increasing, leaf area will raise crop yields under global atmospheric change. Global Change Biol. 23, 1626-1635 (2017).

60. Takai, T. et al. Effects of quantitative trait locus MP3 on number of panicles and rice productivity in nutrient-poor soils of Madagascar. Crop Sci. in press 10.1002/csc2.20344 (2020).

61. Salter, W. T., Merchant, A., Trethowan, R. M., Richards, R. A. \& Buckley, T. N. Wide variation in the suboptimal distribution of photosynthetic capacity in relation to light across genotypes of wheat. AoB Plants 12 plaa039; 10.1093/aobpla/plaa039 (2020).

62. Song, Q., Wang, Y., Qu, M., Ort, D. R. \& Zhu, X.-G. The impact of modifying photosystem antenna size on canopy photosynthetic efficiency-Development of a new canopy photosynthesis model scaling from metabolism to canopy level processes. Plant Cell Environ. 40, 2946-2957 (2017).

63. San, N. S. et al. Differences in lamina joint anatomy cause cultivar differences in leaf inclination angle of rice. Plant Prod. Sci. 21, 302-310; 10.1080/1343943X.2018.1500488 (2018).

64. Yamori, W. Photosynthetic response to fluctuating environments and photoprotective strategies under abiotic stress. J. Plant Res. 129, 379-395 (2016).

65. Taylor, S. H. \& Long, S. P. Slow induction of photosynthesis on shade to sun transitions in wheat may cost at least $21 \%$ of productivity. Phil. Trans. R. Soc. B: Biol. Sci. 372, 1730 (2017).

66. Tanaka, Y., Adachi, S. \& Yamori, W. Natural genetic variation of the photosynthetic induction response to fluctuating light environment. Curr. Opin. Plant Biol. 49, 52-59 (2019).

67. Hirasawa, T. \& Hsiao, T. C. Some characteristics of reduced leaf photosynthesis at midday in maize growing in the field. Field Crops Res. 62, 53-62 (1999).

68. R Core Team. R: A language and environment for statistical computing. https://www.R-project.org/ (2020).

69. Cleveland, W. S. Robust locally weighted regression and smoothing scatterplots. J. Am. Stat. Assoc. 74, 829-836 (1979)

\section{Supplementary Data}

Supplementary Figure S1. Layout of the experimental plots in the paddy field. 
Supplementary Figure S2. Phenotyping procedures. (a) Gas exchange measurement with a MIC-100 closed-type portable photosynthesis system. (b) SPAD measurement with a SPAD-502 chlorophyll meter. (c) Scanning of leaves with a ScanSnap iX1500 document scanner.

Supplementary Figure S3. Dynamics of (a) daily mean temperature and (b) daily mean solar radiation during the growing season. Daily mean temperature was obtained from the Automated Meteorological Data Acquisition System (AMeDAS) Fuchu site $\left(35^{\circ} 41^{\prime} \mathrm{N}, 139^{\circ} 29^{\prime} \mathrm{E}\right)$ and daily mean global solar radiation was obtained from the AMeDAS Tokyo site $\left(35^{\circ} 42^{\prime} \mathrm{N}, 139^{\circ} 45^{\prime} \mathrm{E}\right)$. The points are 5 -day averages. DAT, days after transplanting.

Supplementary Figure S4. Pearson's correlation coefficients of pairs of traits (biomass, crop growth rate, integrated $\mathrm{CO}_{2}$ assimilation rate and mean single leaf area) among all lines examined. Values in bold type are significant $\left(P<0.05\right.$, two-sided $t$-test). Blue, positive correlation; red, negative correlation. AGB $\|_{\text {, }}$ dry weight of aboveground biomass at the second sampling; $\mathrm{CGR}_{\text {Phasel }}$ and CGR Phase II, crop growth rate

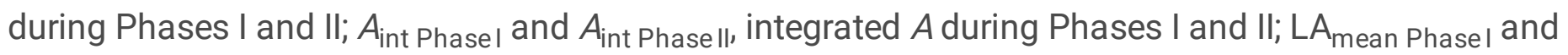
$\mathrm{LA}_{\text {mean Phasell, }}$ mean single leaf area during Phases I and II.

Supplementary Figure S5. Pearson's correlation coefficients of pairs of traits (biomass accumulation, $\mathrm{CO}_{2}$ assimilation rate and other agronomic traits) in (a) Koshihikari lines and (b) Takanari lines during Phase II. Values in bold type are significant $(P<0.05$, two-sided $t$-test). Blue, positive correlation; red, negative correlation. $\mathrm{AGB}_{\|}$, dry weight of aboveground biomass at the second sampling; $\mathrm{CGR}_{\text {Phase ll, }}$ crop growth rate during Phase Il; $A_{\mathrm{max}}$, maximum fitted value of $A ; A_{\mathrm{all}}$, accumulated $A$ during Phase Il; $A_{80}$, accumulated $A$ from 72 days after transplanting (DAT) to $D_{\text {onset; }} A_{\text {dec }}$ accumulated $A$ from $D_{\text {onset }}$ to 128 DAT; $D_{\text {onset }} 1$ day before $A$ declined below $80 \%$ of $A_{\text {max }} ; \mathrm{DTH}$, days to heading; $\mathrm{LA}_{\text {mean Phase II, }}$ mean value of single leaf area during Phase II; SPAD 80 , mean SPAD value before $D_{\text {onset; }}$ SPAD $_{\text {dec }}$, mean SPAD value after $D_{\text {onset }}$

Supplementary Figure S6. Biomass at harvest and crop growth rate (CGR) of Koshihikari, SL1212, Takanari and SL1310 during Phase II. (a) Graphical genotypes of the CSSLs; orange, Koshihikari genomic region; blue, Takanari genomic region. (b) Dry weight of aboveground biomass at the second sampling

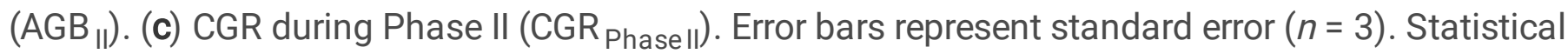
differences were tested by Welch's two-sided $t$-test $(n=3)$.

Supplementary Figure S7. Pearson's correlation coefficients of crop growth rate during Phase II (CGR Phase II) with accumulated $A$ after heading. Accumulated $A$ was the integral from 72 days after transplanting (DAT) to $D_{\text {onset }}\left(1\right.$ day before $A$ declined below $10 \%-90 \%$ of maximum $A$ ). We selected $A_{80}$ because it had the highest correlation in the combined data set and the Takanari line set and the second highest in the Koshihikari line set.

Supplementary Table S1. Standard deviations (SDs) of the traits shown in Figure 2. 
Supplementary dataset. Values of the traits shown in Figures 4 and S5. AGB ${ }_{\|}$, dry weight of aboveground biomass at second sampling; CGR Phase II, crop growth rate during Phase Il; $A_{\text {max }}$ maximum fitted value of $A$ during Phase Il; $A_{\text {all, }}$, accumulated $A$ during Phase Il; $A_{80}$, accumulated $A$ from 72 days after transplanting (DAT) to $D_{\text {onset }} A_{\text {dec }}$, accumulated $A$ from $D_{\text {onset }}$ to 128 DAT; $D_{\text {onset }}, 1$ day before $A$ value declines below $80 \%$ of $A_{\text {max }} ; \mathrm{DTH}$, days to heading; $\mathrm{LA}$ mean Phase I, mean single leaf area during Phase Il; $\operatorname{SPAD}_{80}$, mean SPAD value before $D_{\text {onset }} ; \operatorname{SPAD} D_{\text {dec }}$, mean SPAD value after $D_{\text {onset }}$.

\section{Figures}

Net $\mathrm{CO}_{2}$ assimilation rate $\left(\mu \mathrm{mol} \mathrm{m}{ }^{-2} \mathrm{~s}^{-1}\right)$

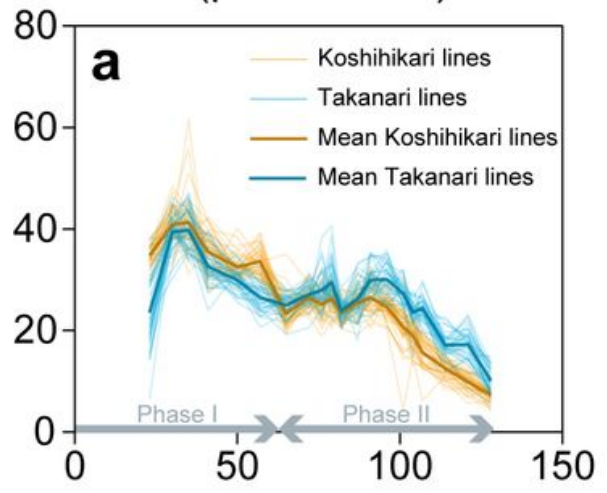

SPAD value

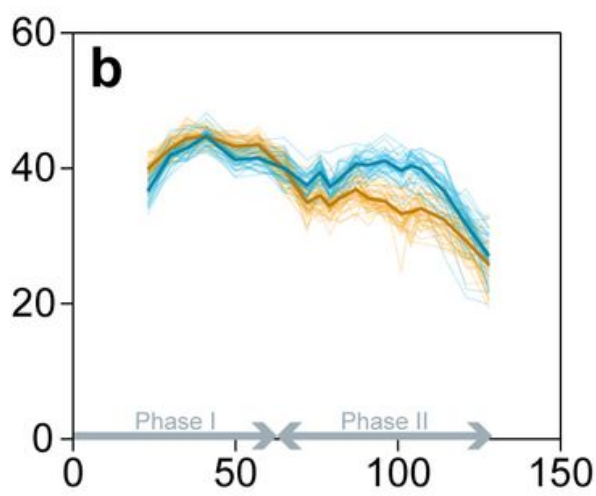

Fig.1 Honda, Ohkubo et al. Single leaf area $\left(\mathrm{cm}^{2}\right)$

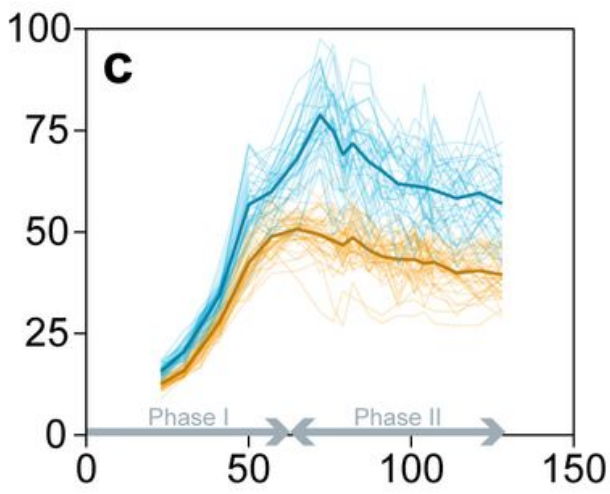

\section{Days after transplanting (day)}

\section{Figure 1}

Dynamics of (a) net CO2 assimilation rate, (b) SPAD value and (c) single leaf area across the entire growing season. Koshihikari lines, Koshihikari-background CSSLs and Koshihikari; Takanari lines, Takanari-background CSSLs and Takanari $(n=3)$. Mean phenotypic values are also shown. Phase I, transplanting to first biomass sampling (71 days after transplanting, DAT); Phase II, first biomass sampling to second biomass sampling (129 DAT). 
Fig.2 Honda, Ohkubo et al.
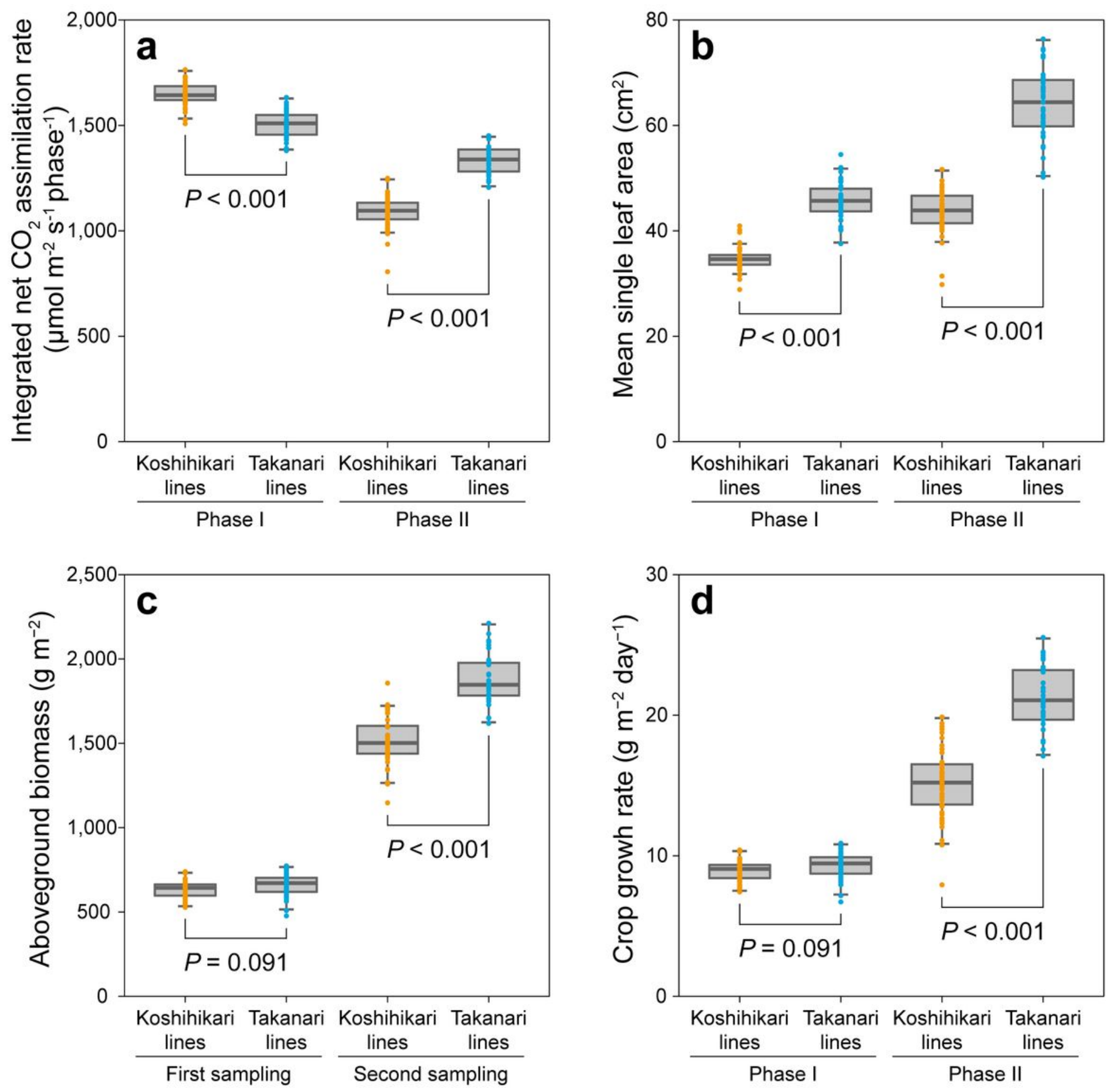

Figure 2

Comparisons of phenotypes between Koshihikari lines and Takanari lines. (a) Integrated net $\mathrm{CO} 2$ assimilation rate, (b) mean single leaf area, (c) dry weight of aboveground biomass, (d) crop growth rate. Abbreviations as in Figure 1. Boxplots: central line, median; boxes, interquartile range (IQR); whiskers, 1.5 × IQR; points, outliers. 
Fig. 3 Honda, Ohkubo et al.
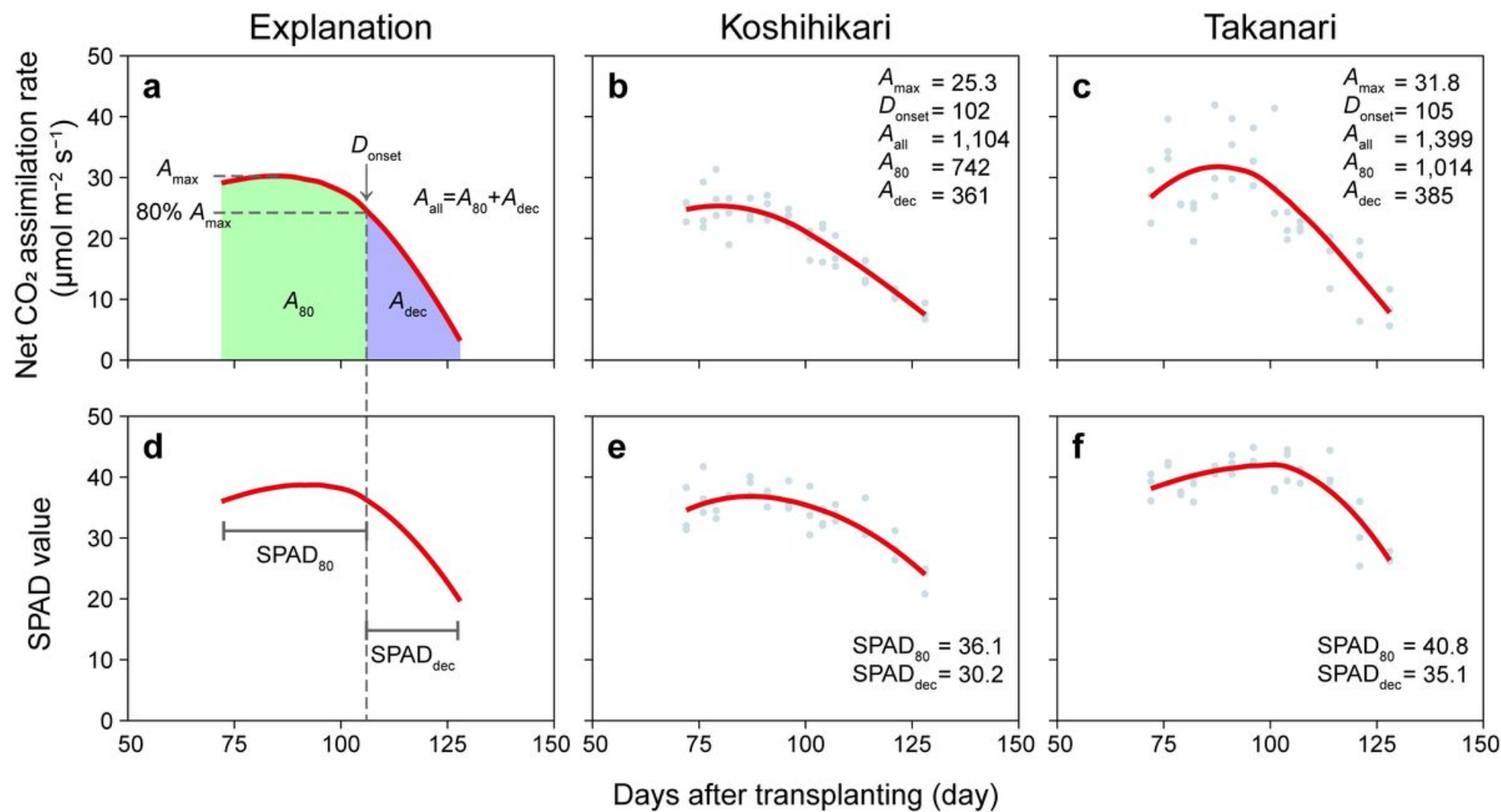

\section{Figure 3}

Curve-smoothing analysis for net $\mathrm{CO} 2$ assimilation rates (A) and SPAD values during Phase II. (a) A curve. Amax, maximum fitted value of A; Donset, 1 day before A declined below $80 \%$ of Amax; Aall, accumulated A during Phase II; A80, accumulated A from 72 DAT to Donset; Adec, accumulated A from Donset to 128 DAT. (b, c) Curve-smoothing analysis of A for (b) Koshihikari and (c) Takanari. Grey points, actual data; red lines, smoothed curves. (d) SPAD curve. SPAD80, mean SPAD value before Donset; SPADdec, mean SPAD value after Donset. $(e, f)$ Curve-smoothing analysis of SPAD for (e) Koshihikari and (f) Takanari. 
Fig.4 Honda, Ohkubo et al.

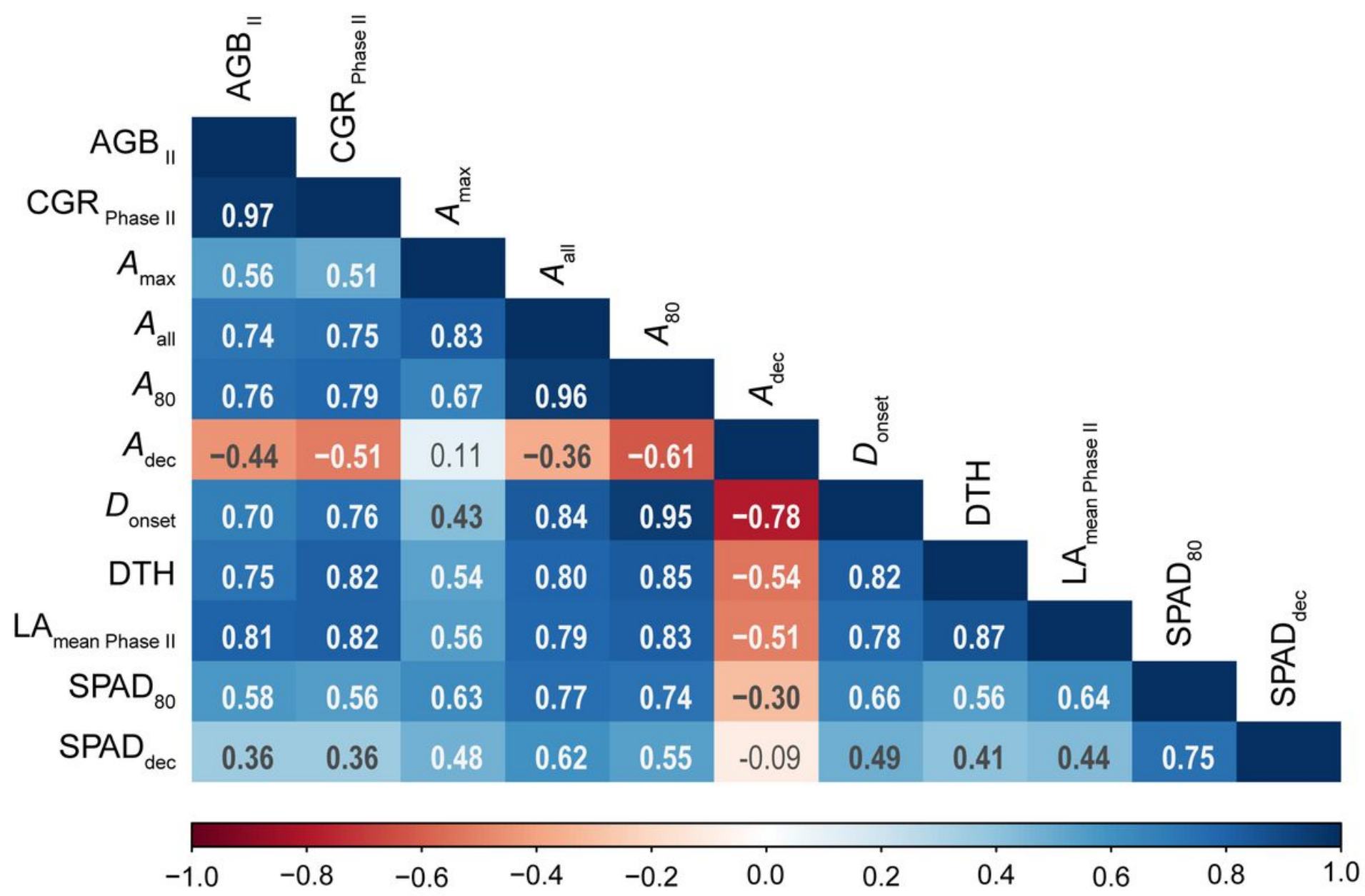

Figure 4

Pearson's correlation coefficients of pairs of traits (biomass accumulation, CO2 assimilation and other agronomic traits) during Phase II among all lines examined. Values in bold type are significant $(P<0.05$, two-sided t-test). Blue, positive correlation; red, negative correlation. AGB II, dry weight of aboveground biomass harvested at 128 DAT; CGR Phase II, crop growth rate during Phase II; DTH, days to heading; LAmean Phase II, mean value of single leaf area during Phase II. Other abbreviations as in Figure 3. 

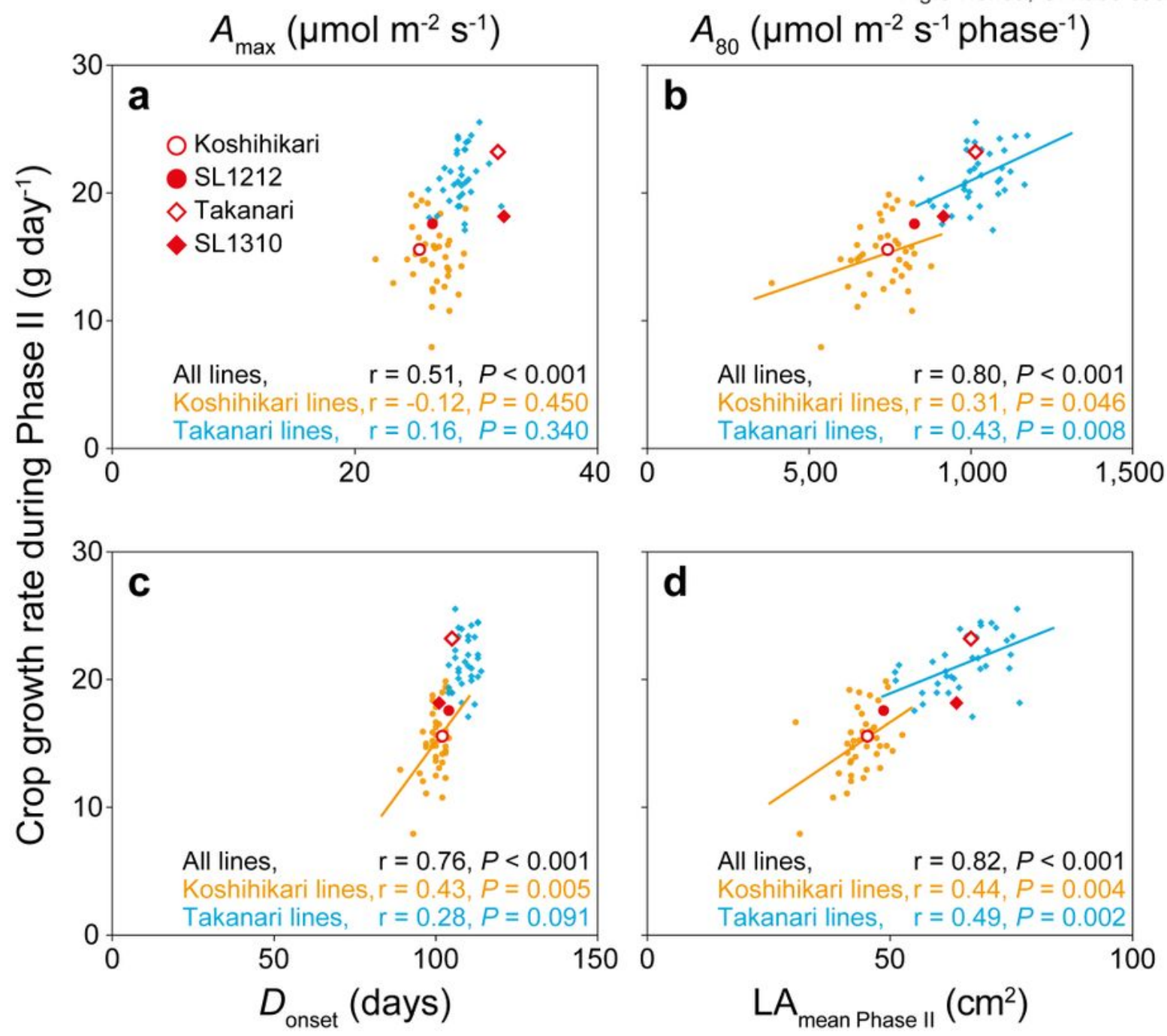

e

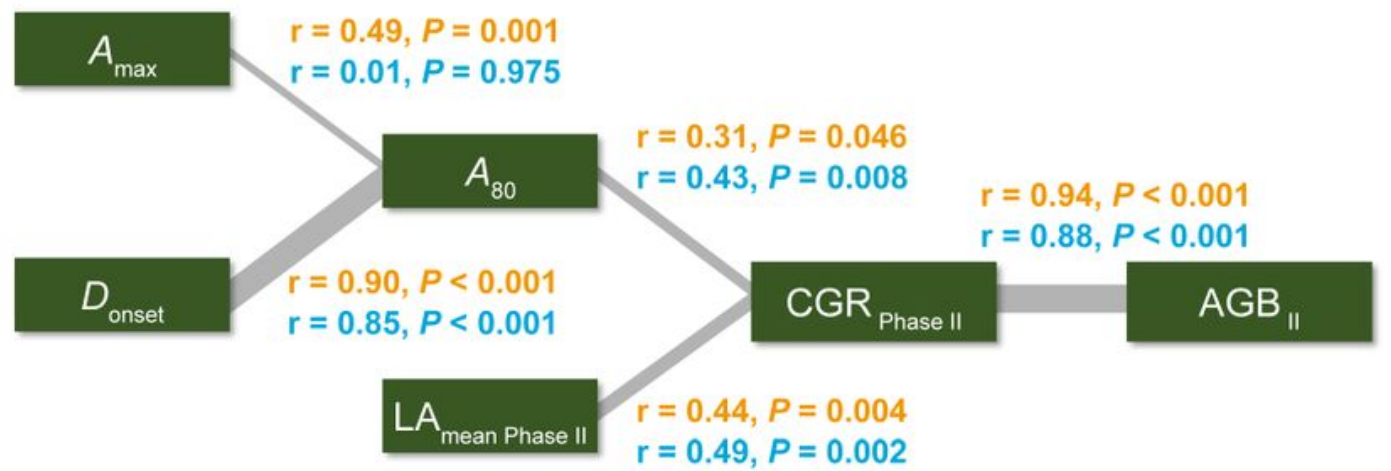

\section{Figure 5}

Relationships between CGR Phase II and (a) Amax, (b) A80, (c) Donset and (d) LAmean Phase II. Orange, Koshihikari lines; blue, Takanari lines. (e) Schematic model showing the factors affecting biomass accumulation. Abbreviations as in Figures 3 and 4.

\section{Supplementary Files}


This is a list of supplementary files associated with this preprint. Click to download.

- Supplementaryinformation.pdf

- Supplementarydataset.xlsx 